\title{
Electronic Agenda Design for a Class with Special Educational Needs
}

\author{
Cárdenas, M.E., Villa, P., Vargas, R. E., Vega, L., Quintero, R.R.
}

Instituto Tecnológico de Culiacán

\begin{abstract}
General population dependency in technological development makes us realize the urgent to develop also applications that take into account people with special educational needs and other capabilities. We present the design of an "electronic agenda" that provides care of special needs of an Orff class at Sinaloa Institute of Culture, whose members are people with different capabilities. Furthermore, we provide a proposal with prototypes in Balsamiq and Kinect.
\end{abstract}

Keywords: Special Education, HumanComputer Interface, Design, Prototyping.

\section{Introduction}

Generally, tasks in a system can be performed by several types of users with very different profiles, which force us to make a careful analysis of task, target user and factors that influence its performance.

Current technologies allow amazing achievements that change our everyday to facilitate the realization of our tasks. Also, the popularity of computer systems in recent years has made our lives increasingly depend of them on different aspects [1].

Based on the above, we have analyzed a special education class at the Sinaloa Institute of Culture (ISIC), in which the range of user needs is extensive.

Below is a brief analysis and description of the method used in the classroom. Also, we present information and obser- vations collected in the field study. We include our design proposal and a brief explanation of some prototypes developed.

\section{Field Study}

We visited the Sinaloa Institute of Culture, where there was a group that receives a special education class based on the Orff method; this is a teaching method that can be used for teaching and music therapy.

Musicology is a recent discipline in music education [2], contributes to the integral development of people through musical awareness of users and improvement in the use of his senses; facilitates muscle control and body, improving development of fine motor and spatial control. All this is an essential part of working with people with disabilities.

The Orff method is an important tool in the development of special education classes because includes activities performed for musical training allowing the job done with music education, and others that allows the development of emotional ties that are important for better integration of these individuals into society [3]. Thus, helps pupils in their integral formation.

Below is a brief description of the class, the individuals comprising it and the techniques used to identify requirements. 


\subsection{Educational Scenario}

The group includes students with special education needs, among which we could detect: low vision, blindness, Down syndrome, attention deficit disorder, autism, hyperactivity, deaf-blindness, deafness, hearing impairment and mobility problems.

The students' ages range between eight and thirty-eight years old. Some students are new in the group, but others have up to four years in it. The way in which students interact in the group made evident their different levels of learning and development skills.

The teacher that leads the class has twenty five years of experience in the field of music and has five years in charge of this class.

During the development of the class activities are carried out with support tactile, visual and sound to achieve multisensory stimulation of students, resulting in an improvement in their social skills, motor skills, memory, learning, coordination and others. The teacher uses music as a mean to integrate activities, applying verbal and nonverbal communication techniques: establish and maintaining eye contact, exaggeration of facial and body gestures and posture and body movements, as well as giving recognition to students for their performance in activities.

\subsection{Techniques applied for Needs Finding}

During the process to discovery needs of this field study we used primarily interview and observation. With the aim of learn from users, during observation we took into account the relations between activities that they perform and skills that are expected to develop [4]. Below is a brief explanation.

We visit the facilities of Sinaloa Institute of Culture, at the classroom assigned to Class Orff, where we observe the interaction between students and the teacher in different sessions, each lasting from two to three hours. During class we took notes of their development and how the objectives were achieved.

After, we held the interview sessions with the teacher to consider her views, needs and expectations for the class, also to analyze the feasibility of implementing changes and integrate new components to the class.

We analyzed the teacher requests, based on the priorities and objectives in the short term, in order to define the order of implementation.

Subsequently, we held several brainstorming sessions, where each team members presented its proposals and subsequently, was analyzed the feasibility of implementing each one of them.

After completing the field study and needs analysis, we developed a design proposal, which is presented below.

\section{Design proposal}

After brainstorming, in which various solutions were proposed to the problems and needs identified, we develop some proposals that could be implemented to improve student achievement and teacher performance.

These proposals were classified into two groups: proposals whose implementation does not require computer technology, when we reported to the teacher, said she would include the method in its work to strengthen the skills and experiences gained in class. The second group consists of the proposals that require different existing computer technologies.

In this paper we present the prototype of an agenda and the proposal chosen among those considered to develop in the second category. 


\section{Early stages of prototyping}

With the aim of developing an agenda that facilitate the development of the class we create several storyboards. These helped us to define interactions that may occur between the user and the system, by setting a detailed sequence of alternatives that are available and their solutions [5].

The storyboards were developed considering the two areas that make the classroom Orff: a traditional area and a theater area. The traditional area has common desks typically arranged in rows and a blackboard at the front wall. The theater area consists basically of an empty space where the teacher incorporates or removes physical resources (such as toys, musical instruments or costumes) for representation and motor development activities. Each area takes half the classroom.

Based on these storyboards, prototypes were developed, first using Balsamiq mockup and then integrating Microsoft Kinect, which is explained briefly in the next section.

\subsection{Prototyping using Balsamiq Mockup}

We developed the prototype using a demo license from Balsamiq Mockup [6]. We developed some system utilities that were consider relevant to the proof of concept.

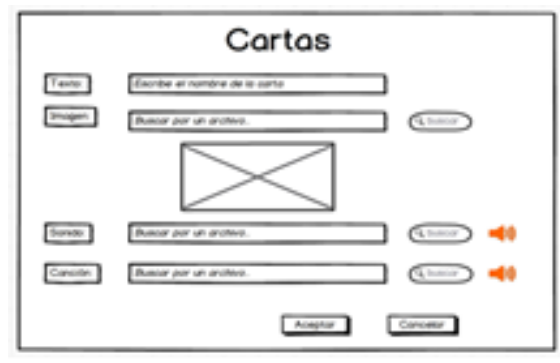

Fig 1: Item registration per category
The resulting agenda consists of several categories, each of which registers cards with an image, image name, its representation in sign language, text and a sound associated with that image. Figure 1 shows the option of registering a new item in a category.

The system can be configured according to the type of user and skill you want to evaluate or develop. For example, if the animal category for deaf students shows an image, the student must write the name of it and make the sign that represents it. In the event that name or sign are not appropriate, it will be informed and asked to proofread. In case of a blind person, a representative sound for the card's item will be activated and the user must enter the name.

Figure 2 shows a card from the Animals Category, which must be recognized by a student based on the image shown, there is an option call "voice" that reproduces the sound emitted for the animal (for blind students) and the option "gesture" that shows its name in sign language (for deaf student).

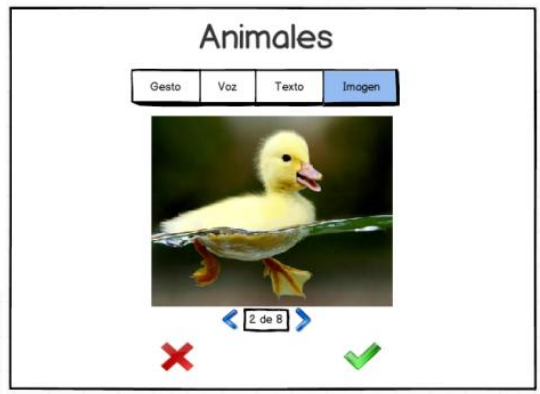

Fig 2: Card Category: Animals

In addition to the examples cited above, a variety of possible configurations may be given, depending on the habilities that you want to develop on the end user. 


\subsection{Prototyping using Kinect}

During prototyping, SDK Kinect libraries were used to facilitate access to the various activities of the agenda [7]. From the beginning, and throughout the execution of the system, gesture recognition can be used to access each of the categories and to interact with the application. Furthermore there are other functionalities. For example, in the song category, student will be informed after each practice if precise gestures need to be improved at certain part of the song. This helps both the parents and the teacher to see the student's progress in motor control.

We develop proof of concept for student's different needs, for example: deaf students have a difficult time to learn a new activity, so when a new activity is available, it will be indicated in sign language (by name, type of activity: song, dance, motor development, etc.). Student will be able to repeat as many times as need those gestures and movements, until he feel satisfied.

\section{Conclusions and Future Work}

We are currently working on the design of an interface that integrates the different components implemented and allows system tuning. To facilitate as much as possible the recognition of sign language, we continue adding agenda's activities to the Kinect platform design.

\section{References}

[1] B. Schneiderman, Designing the user interface: strategies for effective human-computer-interaction, Michigan: Addison Wesley, 1998.

[2] Wikipedia, «Método Orff,» Wikipedia, 0511 2012. [On line]. Available:

http://es.wikipedia.org/wiki/Metodo_ Orff. [Last access: 2811 2012].

[3] P. Sabbatella, «Intervención musical en el alumnado con necesidades educativas especiales: delimitaciones conceptuales desde la pedagogía musical y la musicoterapia,» Universidad de Cádiz, Puerto Real, Cádiz, 2006.

[4] J. R. J. Hackos, «Chapter 2: Thinking about users,» de User and task analysis for interface design, Michigan, Wiley, 1998, pp. 21-50.

[5] L. Galindo Soria, «Un modelo para el desarrollo de sistemas interactivos,» Instituto Politécnico Nacional, México, D.F., 2012.

[6] Balsamiq, «Balsamiq,» Balsamiq, 2012. [On line]. Available: http://www.balsamiq.com/. [Last access: 3011 2012].

[7] M. Corporation, «Kinect for Windows,» Microsoft Corporation, 2013. [On line]. Available: http://www.microsoft.com/enus/kinectforwindows/develop/overvie w.aspx. [Last access: 0904 2013]. 